\title{
Interesting News in Peritoneal Dialysis
}

\author{
An S. De Vriese \\ Renal Unit, AZ Sint-J an AV Brugge and University Hospital Gent, Belgium
}

\section{Introduction}

Long-term PD is characterized by a progressive loss of ultrafiltration capacity. In the majority of cases this decrease in ultrafiltration rate is associated with an elevated transport of small molecular weight solutes [1, 2]. High small solute transport rates result in a rapid absorption of glucose from the peritoneal cavity, leading to an early dissipation of the osmotic gradient and thus a reduced transcapillary ultrafiltration. The transport of small solutes is not influenced by a size-selective restriction barrier but depends essentially on the available vascular surface area. The finding of a high small solute transport rate thus reflects an increased surface area. As it is unlikely that the size of the peritoneum changes, an elevated vascular surface area must be due to an increase of the number of capillaries that contribute to transport, either by recruitment of previously non-perfused capillaries or by formation of new capillaries. It can thus be derived that a stable increase of the transport rates of small solutes points to the presence of peritoneal neoangiogenesis.

Neoangiogenesis has indeed been observed in peritoneal biopsies of patients on long-term PD. A few early reports already mentioned the presence of hypervascularization in the peritoneal membrane of patients on longterm PD [3, 4]. Recently, neovascularization and capillary dilation were reported in peritoneal biopsies and the number of microvessels per area increased along with the duration of PD [5]. Further, an upregulation of endothe- lial nitric oxide synthase, corresponding with an increased vascular density was reported in the peritoneum of longterm PD patients [6]. Another prominent finding in peritoneal biopsies of PD patients is the presence of fibrosis. Variable degrees of expansion of the interstitial matrix and an increased presence of collagen type IV have been described $[3,5]$. Similarly, the vasculature is characterized by fibrosis and subendothelial hyalinization [7, 8], due to an increased deposition of collagen type IV and laminin [7]. The prevalence of this vasculopathy increased with time spent on PD [8].

As outlined above, a pathogenetic link between loss of ultrafiltration capacity and peritoneal neoangiogenesis is a logical assumption [9]. In biopsies from the Peritoneal Biopsy Registry, vessel density was significantly higher in patients with membrane failure [8]. Direct evidence for the etiologic role of neoangiogenesis in the development of ultrafiltration failure has been elegantly provided in an animal model of peritoneal dialysis [10]. Inhibition of neoangiogenesis by gene transfer of angiostatin improved ultrafiltration rate, whereas neutralization of TGF-beta reduced fibrosis but did not affect net ultrafiltration rate [10]. In addition, inhibition of peritoneal neoangiogenesis by anti-VEGF antibodies normalized small solute transport rates in an experimental model of high glucose exposure [11].

The association of the changes in peritoneal function and structure with time spent on PD suggests that they are caused by the chronic exposure of the peritoneal mem-

\begin{tabular}{ll}
\hline KARGER & ( ) 2002 S. Karger AG, Basel \\
Fax +4161306 1234 $34-2766 / 02 / 0924-0763 \$ 18.50 / 0$ \\
$\begin{array}{l}\text { E-Mail karger@karger.ch } \\
\text { www.karger.com }\end{array}$ & $\begin{array}{l}\text { Accessible online at: } \\
\text { www.karger.com/journals/nef }\end{array}$
\end{tabular}

An S. De Vriese

Renal Unit, AZ Sint-Jan AV

Ruddershove, 10

B-8000 Brugge (Belgium)

Tel. +32 504522 02, Fax +32 504522 99, E-Mail an.devriese@azbrugge.be 
brane to the bioincompatible dialysate solutions. The present communication will discuss the dialysate components that are potentially responsible for the peritoneal neoangiogenesis and loss of ultrafiltration capacity. Further, the approaches to prevent the development of these alterations will be reviewed.

\section{Glucose}

The structural alterations observed in the peritoneal membrane of patients on long-term PD are reminiscent of the changes characteristic of diabetic vasculopathy. Hence, the hypothesis has been expounded that the chronic exposure to the high glucose concentrations in peritoneal dialysate is, at least in part, responsible for the development of these changes. Several lines of evidence have supported this contention. In a chronic peritoneal infusion model in the rat, exposure to $3.86 \%$ glucose dialysate caused severe neoangiogenesis and fibrosis of the peritoneal membrane as well as impaired ultrafiltration, whereas infusion of ringers lactate solution produced no major alterations [12]. Patients with peritoneal sclerosis on biopsy have a greater cumulative glucose exposure than controls matched for the duration of PD [5]. In a subgroup of long-term PD patients, a rise in solute transport with time on PD was preceded by an increased peritoneal exposure to hypertonic glucose to compensate for an early loss of residual renal function [13].

VEGF is an endothelial-specific growth factor that plays a prominent role in physiological and pathological angiogenesis [14]. VEGF is up-regulated in diverse cell types cultured in a high glucose environment, as well as in various tissues of experimental animals and humans with diabetes. In view of its known biologic properties and its up-regulation by high ambient glucose concentrations, VEGF is an ideal candidate to provide a mechanistic link between chronic dialysate exposure and peritoneal neoangiogenesis with the associated rise in small solute transport rates and loss of ultrafiltration capacity [15]. We will now review the evidence that supports this hypothesis.

Human peritoneal mesothelial cells recovered from spent dialysate and cultured in vitro have the capacity to produce substantial amounts of VEGF [16]. No correlations were, however, found between supernatant VEGF levels and time on PD, solute transport characteristics, ultrafiltration rate or accumulated dose of glucose [16]. The concentration of VEGF in peritoneal effluent was found to be higher than what could be attributed to transport from the circulation, implying local production [17].
The amount of locally synthesized VEGF correlated with the MTAC of creatinine and urate, glucose absorption and transcapillary ultrafiltration [17]. The local production of VEGF increased with time on PD, although the magnitude of the increase was independent of the initial VEGF levels and the duration of PD at the start of followup [18]. These findings indicate that individual susceptibility to as yet unknown factors may determine the extent of peritoneal VEGF production in response to dialysate exposure. VEGF effluent concentrations decreased after switching to treatment with glucose-free dialysate, with a commensurate effect on the parameters of effective peritoneal surface area [18].

Although these studies present ample circumstantial evidence to incrimate high glucose concentrations in the development of the functional and structural alterations of the peritoneal membrane through the production of VEGF, they do not provide proof of causality. We have used streptozotocin-induced diabetes in the rat as an experimental model for high glucose exposure of the peritoneal membrane [11]. The peritoneal microcirculation in diabetic rats is characterized by a pronounced neoangiogenesis, associated with an increased transport of small solutes. The hyperglycemia-induced microvascular alterations were largely prevented by treatment with a neutralizing monoclonal antibody to VEGF, whereas treatment with an isotype-matched control antibody was without effect (fig. 1). These results are thus the first to support an etiologic role for glucose in the development of peritoneal neoangiogenesis and identify VEGF as an important down-stream mediator [11].

\section{Glucose Degradation Products}

A variety of toxic glucose degradation products (GDPs) are formed during heat-sterilization of peritoneal dialysate and to a lesser extent during prolonged storage. Formaldehyde, acetaldehyde, 2-furaldehyde, 5-hydroxymethylfurfural, glyoxal, methylglyoxal and 3-deoxyglucosone are the best characterized GDPs, but undoubtedly many other, as yet unknown compounds are generated.

Cultured rat mesothelial and human endothelial cells expressed VEGF in response to methylglyoxal, but not to glyoxal or 3-deoxyglucosone [19]. Intraperitoneal exposure to methylglyoxal increased VEGF expression in peritoneal tissue of experimental animals [19]. Although these results clearly need confirmation, they suggest that GDPs may contribute to peritoneal neoangiogenesis by augmenting local production of VEGF. 
Fig. 1. Microvascular density was significantly increased in the peritoneal membrane of diabetic rats. The neoangiogenesis was prevented by chronic treatment with antiVEGF antibodies $(\mathrm{Ab})$, but not by control Ab. VEGF blockade did not decrease vascular density in control rats. $* \mathrm{p}<0.01$ vs. controls, $* * \mathrm{p}<0.01$ vs. diabetes and diabetes + control Ab. From De Vriese et al. [11], with permission.

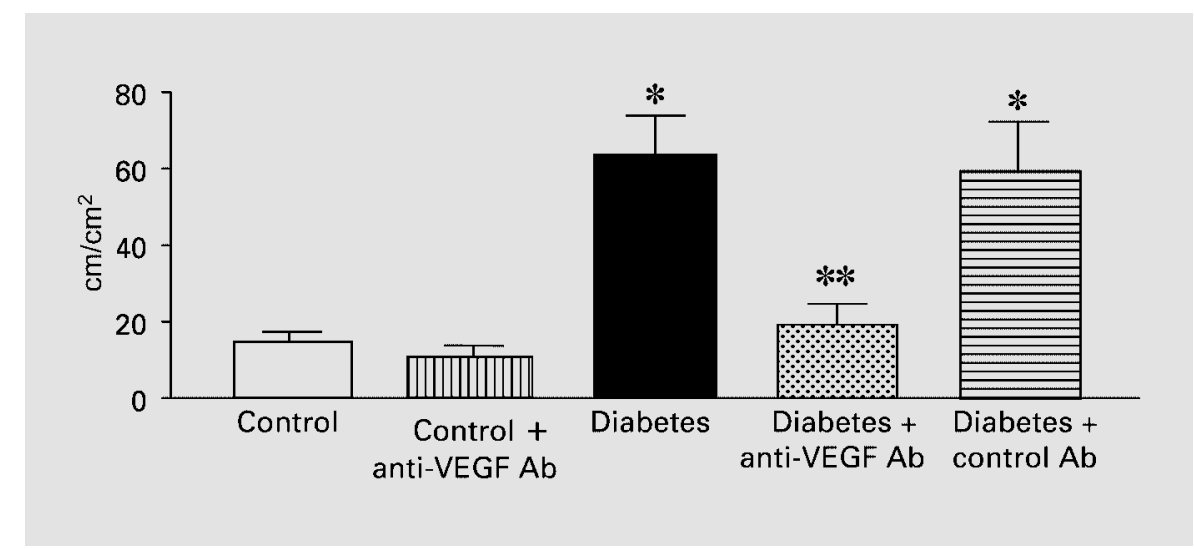

Recently, we have reported that peritoneal dialysate exerts important vasoactive effects [20]. Exposure to conventional dialysate caused a dramatic increase in peritoneal arteriolar flow resulting in a recruitment of previously non-perfused capillaries. Dialysates with low GDP levels had only minor hemodynamic effects, while resterilization of these dialysates to increase GDP content induced similar vasoreactivity as caused by the conventional dialysate (fig. 2). The results thus strongly suggest an important etiologic role for GDPs in the dialysateinduced vasoreactivity. The expansion of the effective peritoneal vascular surface area resulting from the capillary recruitment may be an additional cause of decreased ultrafiltration capacity.

\section{Advanced Glycation and End Products}

Glucose and a variety of other reactive carbonyl compounds are known to bind nonenzymatically to free amino groups on proteins or to lipids. Through a series of oxidative and nonoxidative reactions, advanced glycation end products (AGEs) are formed irreversibly. AGE formation occurs during normal aging, but is accelerated when the concentrations of the substrate molecules are increased and when the prevailing oxidant stress is high. The peritoneum is chronically exposed to high glucose concentrations, vastly in excess of those found in diabetes, and large amounts of glucose are continuously absorbed across the peritoneal membrane. The uremic environment is associated with a high oxidative stress, thus further promoting generation of AGEs. Breakdown of AGEmodified tissue proteins yields low molecular weight AGEs, which are normally cleared through the kidney [21]. As residual renal function deteriorates, the plasma concentration of these low molecular weight AGEs rises, while they retain their biological activity. In accordance, increased levels of AGEs have been found in the serum of PD patients [22]. Finally, the presence of GPDs in peritoneal dialysate facilitates the formation of AGEs [23, 24]. Taken together, in the peritoneal cavity of PD patients, conditions are optimal for a dramatically accelerated AGE formation and accumulation.

In vitro studies have demonstrated that AGE formation indeed occurs in PD fluid at a rate higher than what can be explained by the glucose concentration alone [25]. AGEs have been detected immunocytochemically in the mesothelium, submesothelial stroma and vascular wall of PD patients [6, 26-28]. The degree of AGE-accumulation correlated with the time spent on PD [26, 28].

Recently, glycated serum albumin was shown to promote VEGF expression in cultured human peritoneal mesothelial cells [29]. AGEs may thus present a third pathway leading to peritoneal neoangiogenesis (fig. 3). VEGF and carboxymethyllysine [19] or pentosidine [6] were found to co-localize in the mesothelial layer and the vascular endothelium of the peritoneal membrane of PD patients, but these findings are purely descriptive and do not demonstrate causality.

Attempts have been made to correlate the extent of AGE accumulation with functional parameters [26-28]. Peritoneal staining for AGEs increased along with time on PD and was associated with a higher permeability to various solutes [26, 28]. In another study, the degree of interstitial fibrosis and vascular sclerosis correlated with interstitial and vascular AGE accumulation, respectively [27]. An inverse correlation was found between these peritoneal histological changes and ultrafiltration volume [27]. The results incriminate AGE accumulation in the pathophysiology of ultrafiltration failure, although the precise 

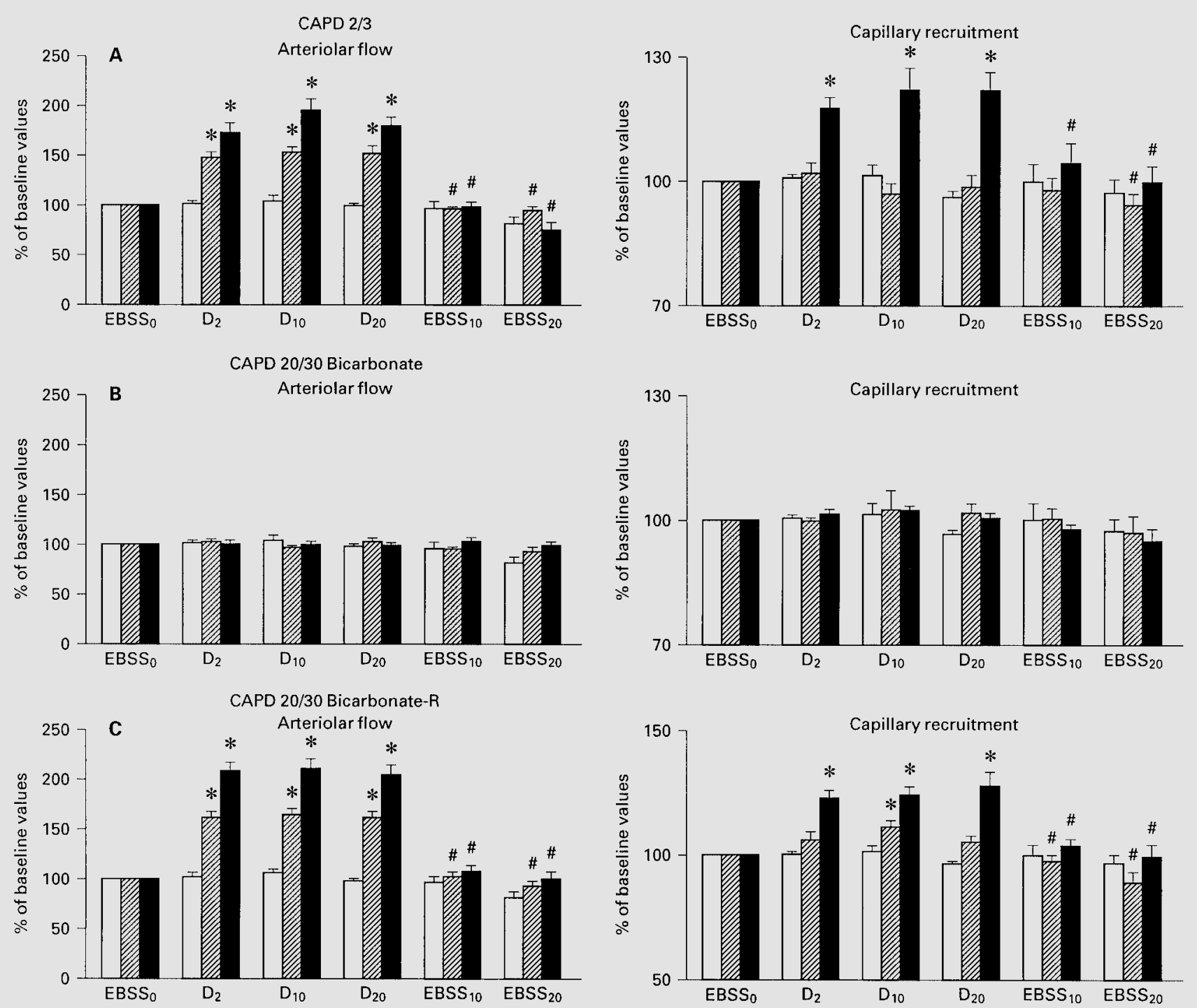

Fig. 2. Percentage change of arteriolar flow or perfused capillary length per area after superfusion with Earle's balanced salt solution (EBSS) (open bars), dialysate with $1.5 \%$ glucose (hatched bars) or dialysate with $4.25 \%$ glucose (closed bars). Measurements were made before exposure to dialysate $\left(\mathrm{EBSS}_{0}\right), 2 \mathrm{~min}$ after dialysate $\left(\mathrm{D}_{2}\right)$, $10 \mathrm{~min}$ after dialysate $\left(\mathrm{D}_{10}\right), 20 \mathrm{~min}$ after dialysate $\left(\mathrm{D}_{20}\right), 10 \mathrm{~min}$ after withdrawal of dialysate $\left(\mathrm{EBSS}_{10}\right)$ and $20 \mathrm{~min}$ after withdrawal of dialysate $\left(\mathrm{EBSS}_{20}\right)$. A Exposure to conventional acidic lactate dialysate (CAPD 2/3) dramatically increased arteriolar flow in the perito-

neal membrane $\left({ }^{*} \mathrm{p}<0.0001\right.$ vs. $\mathrm{EBSS}_{0},{ }^{\#} \mathrm{p}<0.0001$ vs. $\left.\mathrm{D}_{20}\right)$ and recruited additional capillaries for perfusion $\left(* \mathrm{p}<0.05\right.$ vs. EBSS $_{0}$, $\# \mathrm{p}<0.04$ vs. $\mathrm{D}_{20}$ ). B A neutral bicarbonate-buffered dialysate with low GDP content (CAPD 20/30 bicarbonate) was devoid of hemodynamic effects. C Resterilization of CAPD 20/30 bicarbonate to increase the GDP content caused an increase in ateriolar flow $\left(^{*} \mathrm{p}<\right.$ 0.00001 vs. $\mathrm{EBSS}_{0},{ }^{\#} \mathrm{p}<0.0001$ vs. $\mathrm{D}_{20}$ ) and capillary recruitment $\left(* \mathrm{p}<0.01\right.$ vs. $\mathrm{EBSS}_{0},{ }^{\#} \mathrm{p}<0.05$ vs. $\left.\mathrm{D}_{20}\right)$ that was similar to that of conventional dialysate. From Mortier et al. [20], with permission. 


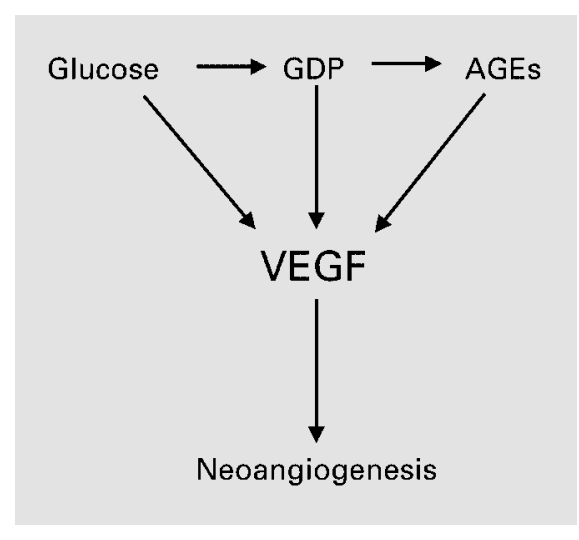

Fig. 3. VEGF as a mediator of glucotoxicity to the peritoneal membrane.

mechanisms underlying this association remain unclear. It is generally acknowledged that the interstitium does not contribute importantly to the barrier function of the peritoneal membrane. It is unknown whether interstitial fibrosis can be associated with a decreased hydraulic permeability or with a more rapid transport of small solutes. On the other hand, a correlation has been reported between the degree of interstitial fibrosis and vascular density in the membrane of long-term PD patients [5]. It may be more logical to postulate that peritoneal AGE accumulation is associated with both interstitial fibrosis and neoangiogenesis, while the latter phenomenon is primarily responsible for the increased small solute transport and loss of ultrafiltration capacity. Unfortunately, no morphometrical analysis of vascular density was performed in the studies on peritoneal AGE accumulation [26-28].

\section{Therapeutic Approaches}

Any therapeutic strategy that will reduce glucose and GDP exposure with the resultant AGE accumulation in the peritoneal membrane, has the potential to prevent the development of ultrafiltration failure. Several non-glucose-based dialysates are currently used, including icodextrin, glycerol and amino acids. In addition, dialysates with low GDP content, due to separate sterilization of glucose at very low $\mathrm{pH}$, have become available. Extensive in vitro testing has suggested that these new dialysates my be more biocompatible than conventional dialysates. Their effects on in vivo peritoneal membrane function are currently being investigated.
Heat-sterilized icodextrin dialysate results in less glycation and AGE formation in vitro as compared to conventional heat-sterilized glucose-based dialysate [21, 30]. In keeping with these findings, less AGE-specific immunostaining was observed in the peritoneum of animals dialyzed with icodextrin than in those treated with conventional dialysate [31]. A switch to icodextrin- and glycerol-based dialysis in small groups of patients with severe UFF resulted in decreased pentosidine dialysate levels, as well as in some improvement of ultrafiltration [12]. A randomized trial in continuous cycling PD patients with a follow-up of 2 years showed, however, no difference in peritoneal transport characteristics and peritoneal membrane markers between the icodextrin and standard dialysate groups [32].

In vitro AGE formation was decreased in doublechamber dialysate solutions with low GDP content [33]. In accordance, lower AGE-staining in the peritoneal membrane and a better ultrafiltration [34], as well as less pronounced submesothelial thickening [35], were reported in rats exposed to low GDP dialysate as compared to conventional dialysate. Finally, neoangiogenesis was significantly decreased in the peritoneal membrane of rats that were long-term exposed to a bicarbonate/lactate buffered dialysate with low GDP content as compared to standard dialysate [36].

\section{Conclusion}

Although the evidence is at times circumstantial, the following hypothesis can be derived from the available data. The high glucose concentrations present in conventional peritoneal dialysate and the formation of toxic GDPs are associated with the accumulation of AGEs in the peritoneal membrane. Glucose, GDPs and AGEs all increase the expression of VEGF in the peritoneum, most probably in mesothelial cells. VEGF induces neoangiogenesis in the peritoneal microcirculation, thereby increasing the effective vascular surface area. The resultant rise in the small solute transport rates leads to a rapid dissipation of the osmotic gradient and a loss of ultrafiltration capacity. Ultimate proof for this hypothesis will come from the demonstration that new dialysates, nonglucose-based or with low GDP content, better preserve long-term peritoneal ultrafiltration capacity. Such trials are currently underway and the results are eagerly awaited. 


\section{Discussion}

Bommer: You showed us the presence of neoangiogenesis and an increased vascular bed after exposure to high glucose concentrations. How was the solute transport rate per $\mathrm{mm}^{2}$ vessel? In other words, is the permeability of the vessel itself increased or is the transport capacity increased because of a higher number of capillaries?

De Vriese: The transport of small solutes is fundamentally dependent on the vascular surface area that is available for transport, and is not determined by the intrinsic permeability of the membrane. Therefore, the findings of increased small solute transport rates only allow us to make conclusions about the density of the peritoneal vascular network. In contrast, the transport of macromolecules is determined by the intrinsic permeability of the membrane. By administering a fluorescent macromolecule, we have demonstrated a rapid leakage of this molecule into the perivascular interstitium in experimental animals exposed to high glucose concentrations, suggesting that the intrinsic permeability of the vessels is also increased [11].

Ritz: After your beautiful studies there is no doubt that VEGF is important. But, if you expose tissue to VEGF it is only the capillaries that are expressed. In order to have a structured neoformation of vasculature, you need other angiogenetic factors. Do you know what other vascular agonists are doing in this model? And second, we know that there are different isoforms in the VEGF-family. It would be naive to assume that there is only one VEGF isoform which is responsible. Do you know which other peptide of VEGF is relevant in this context?

De Vriese: I will first answer the latter part of your question. The VEGF family consists of several isoforms that are generated as a result of alternative splicing from the VEGF gene. The isoforms have different biological properties such as the ability to bind to cellsurface proteoglycans. It is logical to assume that these isoforms also have different biological effects, but as yet very little is known about it. For our studies, we have used neutralizing antibodies that were raised against $\mathrm{VEGF}_{165}$, which is the predominant splice form for most cell types [11].

Concerning the first part of your question, neoangiogenesis and formation of a structured vascular network requires a complex interplay between different growth factors. Our studies have demonstrated that VEGF plays a pivotal role in glucose-induced peritoneal neoangiogenesis, since neutralization of VEGF abolishes the neoangiogenetic response to a high glucose environment. The results certainly do not exclude a role for other growth factors. Recently, overexpression of TGF-beta in the peritoneal membrane was shown to be associated with fibrosis and neoangiogenesis [37]. However, TGFbeta has the capacity to up-regulate VEGF expression [38]. It therefore remains unclear whether TGF-beta causes neoangiogenesis by itself or through VEGF as a down-stream mediator.

Ritz: One elegant way to prove your hypothesis is to use VEGF knockouts. This would certainly prove that VEGF is the crucial factor for neoangiogenesis in this particular model. I think VEGF is even more important than what is apparent from your plots. Exposure of the peritoneal membrane to glucose degradation products will almost certainly increase oxidative stress, and this will scavenge NO. This should impede vasodilatation. Do you have information on iNOS or eNOS-expression?

De Vriese: We have found that the expression of eNOS is upregulated in the peritoneal membrane of experimental animals that are exposed to high glucose concentrations [Stoenoui and De Vriese, unpubl. observations]. This is primarily a consequence of the presence of an increased endothelial surface area. However, it appears that also the relative eNOS expression, i.e. the expression of eNOS per endothelial cell, is increased. It is possible that this is, as you suggest, due to an increased scavenging of NO, requiring an up-regulation of the NO production. However, I believe it is rather evidence of ongoing angiogenesis, as $\mathrm{NO}$ is a known mediator of angiogenesis [39-41]. There is more and more evidence that the characteristic biological actions of VEGF, i.e. endothelium-dependent vasodilation, microvascular hyperpermeability and neoangiogenesis, are mediated by NO. Indeed, neutralization of VEGF prevented the up-regulation of eNOS in the peritoneal membrane in our model [Stoenoui and De Vriese, unpubl. observations], indicating that it is a VEGF-dependent phenomenon.

Andrassy: Normally, AGE is counteracted by RAGE production. Do you know the details of the RAGE (AGE-receptor) and are there new tools which act against RAGE? Are these compounds downregulated during the exposure of the peritoneum membrane to dialysate? What is known about this?

De Vriese: That's an excellent point. Part of the pathogenicity of AGEs is related to the interaction with their cellular receptors, the RAGEs, which results in sustained cellular activation and an increase of the oxidative stress. Uremia up-regulates the expression of RAGE on endothelial cells $[42,43]$. The presence of RAGE has also been documented on mesothelial cells [44]. The binding of AGE to the RAGE on mesothelial cells resulted in an upregulation of the VCAM1 expression [44]. The effect of the AGE-RAGE interaction on peritoneal pathophysiology during PD could be studied by the administration of agents that interfere with this interaction, e.g. neutralizing antibodies or recombinant RAGE. However, no such studies have been published as yet.

Zeier: Could I come back to the dialysis solutions? One successful progress in the development of dialysis solutions is the prevention of GDP formation in the more-compartment bags. On the other hand, clinical studies have been done consorting different buffers in the CAPD solution. My question is, would you either focus on the low GDP bags or on specific buffer-containing solution?

De Vriese: Conventional dialysate is a very unphysiologic solution, due to the acidic $\mathrm{pH}$, the hyperosmolality, the high glucose and lactate concentrations and the presence of glucose degradation products. It profoundly affects different aspects of peritoneal membrane homeostasis, including host defense, angiogenesis and extracellular matrix synthesis. We have just started to unravel which dialysate component is responsible for which adverse effect. Acidic $\mathrm{pH}$, lactate and GDPs all play a role and there is probably a complex interaction between these different factors.

Zeier: Let me come back to the GDPs. Do you have experience that low GDP solutions better preserve residual renal function in chronic CAPD? This is in my opinion an important issue because the residual renal function is an extraordinary important part of adequacy in CAPD.

De Vriese: In two short-term studies, residual renal function, as estimated by the average of the renal urea and creatinine clearance, was not different after treatment with a bicarbonate- or a bicarbonate/lactate-buffered solution with low GDP content as compared to conventional dialysate $[45,46]$. Residual renal function was, however, not a primary end point of these studies. To validly study this aspect, one probably needs longer observation periods and more accurate measures of residual renal function. 
Schwenger: You mentioned that high glucose exposure induces VEGF expression. Did you measure the influence of different $\mathrm{pH}$ values on VEGF formation?

De Vriese: Our studies were performed in vivo, using diabetes as a model for a high ambient glucose environment in the peritoneal cavity. The experimental set-up therefore did not allow us to manipulate $\mathrm{pH}$. An important stimulus for VEGF expression is tissue hypoxia. It is therefore not unlikely that acidic $\mathrm{pH}$ has a promoting effect on VEGF expression. I am, however, unaware of any data on the combined effect of acidity and high glucose concentrations on VEGF expression.

Zeier: Fibrosis of the peritoneum will not regress after renal transplantation. But how about the clearance of AGE products in the peritoneum following renal transplantation? Are there any data, e.g. biopsy studies?

De Vriese: To my knowledge there are no data, but we can speculate on what might happen. Low molecular weight AGEs that are formed through breakdown of AGE-modified tissue proteins are normally cleared in the urine [22]. In accordance, uremic patients have increased serum levels of AGEs. Restoration of renal function after transplantation will decrease the circulating levels of AGEs. Low molecular AGEs that are transported from the peritoneal cavity into the systemic circulation might thus be cleared. In addition, after withdrawal of PD the peritoneal membrane is no longer exposed to high glucose concentrations. Thus, there is no longer a substrate for new AGE formation. However, AGEs are known to accumulate in tissues and form cross-links. During this process oxygen-derived free radicals are generated. Once the initial glycation reaction has taken place, the formation of cross-links continues, even in the absence of substrate. So, whether the AGE-accumulation in the peritoneal membrane will regress after transplantation probably depends on the balance between these different processes.

Schwenger: What do you think about GDPs and AGE: is this only a local peritoneal problem or do you think that this is a systemic issue? In other words would you expect a certain amount of resorption?

De Vriese: Glycated albumin levels were found to be higher in dialysate than in serum [47]. They could thus be transported across the peritoneal membrane and contribute to the systemic burden of AGEs. In addition, the vast quantities of glucose that are resorbed during PD could be a substrate for systemic AGE-formation. Paradoxically, the serum levels of pentosidine were lower in PD patients than in hemodialysis patients [22], suggesting rather that there is transport from the serum into the dialysate. However, AGEs are a very heterogeneous group of compounds and the kinetics might be quite different for different AGEs.

Schwenger: In your rat model did you measure for example VEGF in the circulation after the absorption of GDPs?

De Vriese: Measurement of systemic VEGF levels is fraught with technical problems. The free circulating VEGF levels are very low. VEGF is present in the thrombocytes and is released upon coagulation of the blood. This phenomenon makes it very difficult to correctly measure systemic VEGF levels. In addition, VEGF is a paracrine mediator and systemic levels probably give little information about what is happening locally in the tissues.

Ritz: May I come back to the question of chronic sclerosing peritonitis. We know of course that this was attributed to the buffer. But it may be worthwhile to look into the problem again. The buffer capacities of lactate and acetate are quite different. Do you have still material from such cases? Did you observe such cases of sclerosing peritonitis? The reason why I'm asking: it is quite possible that during sterilisation in low buffer systems there may be more glucose degradation products in the former CAPD dialysis solution as compared to the lactate solutions. And therefore the problem is not the buffer but the glucose degradation products. It would be interesting to look by immune staining at peritoneum material which might be still preserved from these cases which may be still available in some units.

De Vriese: The time-, temperature- and $\mathrm{pH}$-dependence of GDPformation is well known [48]. However, I am unaware of data on the effect of different buffers on the rate of GDP formation. In the new more-chamber bags, the buffer is kept separated from the glucose. The buffer choice will therefore not interfere with GDP formation.

Zeier: What is your opinion on diabetic patients on CAPD? Do you have any information on diabetic experimental animals on CAPD?

De Vriese: Is your question whether the functional and structural alterations of the peritoneal membrane upon dialysate exposure develop more rapidly in diabetic patients than in nondiabetic patients?

Zeier: Yes.

De Vriese: Studies comparing diabetic and non-diabetic patients on PD have yielded conflicting results, mostly because of low patient numbers and the heterogeneity of the population. Few studies are available on the morphology of the peritoneal membrane in diabetic patients. The mesothelial basement membrane had a normal morphology in both nondiabetic and diabetic patients before the start of PD and showed similar replication in both groups of patients after several months of PD [49, 50]. The basal lamina of stromal blood vessels was normal before treatment in nondiabetic patients, but reduplicated after some time on PD. In diabetic patients, the reduplication of the capillary basement membrane was already present in the initial biopsy and aggravated rapidly upon exposure to dialysate $[49,50]$. Functionally, diabetic patients tended to start PD with higher small solute transport rates $[2,28]$. Conversely, a significantly increased number of patients with type I and II diabetes was found in the patient group with high transport rates [51]. Several authors found that initial differences in transport characteristics between diabetic and nondiabetic patients disappeared after long-term PD [28, 52]. Thus, although the evidence is limited, the peritoneal membrane in diabetic patients does not appear to deteriorate more rapidly than in nondiabetic patients. 


\section{References}

1 Davies SJ, Bryan J, Phillips L, Russell GI: Longitudinal changes in peritoneal kinetics: the effects of peritoneal dialysis and peritonitis. Nephrol Dial Transplant 1996;11:498-506.

2 Selgas R, Fernandez-Reyes MJ, Bosque E, Bajo MA, Borrego F, Jimenez C, Del Peso G, De Alvaro F: Functional longevity of the human peritoneum: How long is continuous peritoneal dialysis possible? Results of a prospective medium long-term study. Am J Kidney Dis 1994; 23:64-73.

3 Rubin J, Herrera GA, Collins D: An autopsy study of the peritoneal cavity from patients on continuous ambulatory peritoneal dialysis. Am J Kidney Dis 1991;18:97-102.

4 Verger C, Brunschvicg O, Le Charpentier Y, Lavergne A, Vantelon J: Structural and ultrastructural peritoneal membrane changes and permeability alterations during continuous ambulatory peritoneal dialysis. Proc Eur Dial Transplant Assoc 1981;18:199-205.

5 Mateijsen MA, van der Wal AC, Hendriks PM, Zweers MM, Mulder J, Struijk DG, Krediet RT: Vascular and interstitial changes in the peritoneum of CAPD patients with peritoneal sclerosis. Perit Dial Int 1999;19:517-525.

6 Combet S, Miyata T, Moulin P, Pouthier D, Goffin E, Devuyst O: Vascular proliferation and enhanced expression of endothelial nitric oxide synthase in human peritoneum exposed to long-term peritoneal dialysis. J Am Soc Nephrol 2000; 11:717-728.

7 Honda K, Nitta K, Horita S, Yumura W, Nihei $\mathrm{H}$ : Morphological changes in the peritoneal vasculature of patients on CAPD with ultrafiltration failure. Nephron 1996;72:171-176.

8 Williams JD, Craig KJ, Topley N, Von Ruhland C, Fallon M, Newman GR, Mackenzie RK, Williams GT: Peritoneal Biopsy Study Group: Morphologic changes in the peritoneal membrane of patients with renal disease. J Am Soc Nephrol 2002;13:470-479.

9 De Vriese AS, Mortier S, Lameire NH: Neoangiogenesis in the peritoneal membrane: Does it play a role in ultrafiltration failure? Nephrol Dial Transplant 2001;16:2143-2145.

10 Margetts PJ, Gyorffy S, Klob M, Yu L, Hoff $\mathrm{CM}$, Holmes CJ, Gauldie J: Antiangiogenic and antifibrotic gene therapy in a chronic infusion model of peritoneal dialysis in rats. $\mathbf{J}$ Am Soc Nephrol 2002;13:721-728.

11 De Vriese AS, Tilton RG, Stephan CC, Lamaire N: Diabetes-induced microvascular proliferation and hyperpermeability in the peritoneum: Role of vascular endothelial growth factor. J Am Soc Nephrol 2001;12:1734-1741.

12 Krediet RT, Zweers MM, van der Wal AC, Struijk DG: Neoangiogenesis in the peritoneal membrane. Perit Dial Int 2000;20(suppl 2):S19-25.

13 Davies SJ, Phillips L, Naish PF, Russell GI: Peritoneal glucose exposure and changes in membrane solute transport with time on peritoneal dialysis. J Am Soc Nephrol 2001;12: 1046-1051.
14 Neufeld G, Cohen T, Gengrinovitch S, Poltorak Z: Vascular endothelial growth factor and its receptors. FASEB J 1999;13:9-22.

15 De Vriese AS, Mortier S, Lameire NH: Glucotoxicity of the peritoneal membrane: The case for VEGF. Nephrol Dial Transplant 2001;16: 2299-2302.

16 Selgas R, del Peso G, Bajo MA, Castro MA, Molina S, Cirugeda A, Sanchez-Tomero JA, Castro MJ, Alvarez V, Corbi A, Vara F: Spontaneous VEGF production by cultured peritoneal mesothelial cells from patients on peritoneal dialysis. Perit Dial Int 2000;20:798-801.

17 Zweers MM, de Waart DR, Smit W, Struijk DG, Krediet RT: Growth factors VEGF and TGF-beta1 in peritoneal dialysis. J Lab Clin Med 1999;134:124-132.

18 Zweers MM, Struijk DG, Smit W, Krediet RT: Vascular endothelial growth factor in peritoneal dialysis: A longitudinal follow-up. J Lab Clin Med 2001;137:125-132.

19 Inagi R, Miyata T, Yamamoto T, Suzuki D, Urakami K, Saito A, van Ypersele de Strihou C, Kurokawa K: Glucose degradation product methylglyoxal enhances the production of vascular endothelial growth factor in peritoneal cells: Role in the functional and morphological alterations of peritoneal membranes in peritoneal dialysis. FEBS Lett 1999;463:260-264.

20 Mortier S, De Vriese AS, Schaub TS, Van de Voorde J, Passlick-Deetjen J, Lameire NH: Hemodynamic effects of conventional and new peritoneal dialysis solutions on the microcirculation of the rat peritoneal membrane: Role of acidity, buffer choice, glucose concentration and glucose degradation products. J Am Soc Nephrol 2002;13:480-489.

21 Dawnay AB, Millar DJ: Glycation and advanced glycation end-product formation with icodextrin and dextrose. Perit Dial Int 1997; 17:52-58.

22 Friedlander MA, Wu YC, Schulak JA, Monnier VM, Hricik DA: Influence of dialysis modality on plasma and tissue concentrations of pentosidine in patients with end-stage renal disease. Am J Kidney Dis 1995; 25:441-451.

23 Linden T, Forsback G, Deppisch R, Henle T, Wieslander A: 3-Deoxyglucosone, a promoter of advanced glycation end products in fluids for peritoneal dialysis. Perit Dial Int 1998;18: 290-293.

24 Schalkwijk CG, Posthuma N, ten Brink HJ, ter Wee PM, Teerlink T: Induction of 1,2-dicarbonyl compounds, intermediates in the formation of advanced glycation end-products, during heat-sterilization of glucose-based peritoneal dialysis fluids. Perit Dial Int 1999;19:325333.

25 Lamb EJ, Cattell WR, Dawnay AB: In vitro formation of advanced glycation end products in peritoneal dialysis fluid. Kidney Int 1995; 47:1768-1774.
26 Nakayama M, Kawaguchi Y, Yamada K, Hasegawa T, Takazoe K, Katoh N, Hayakawa H, Osaka N, Yamamoto H, Ogawa A, Kubo H, Shigematsu T, Sakai O, Horiuchi S: Immunohistochemical detection of advanced glycosylation end-products in the peritoneum and its possible pathophysiological role in CAPD. Kidney Int 1997;51:182-186.

27 Honda K, Nitta K, Horita S, Yumura W, Nihei H, Nagai R, Ikeda K, Horiuchi S: Accumulation of advanced glycation end products in the peritoneal vasculature of continuous ambulatory peritoneal dialysis patients with low ultrafiltration. Nephrol Dial Transplant 1999;14: 1541-1549

28 Park MS, Lee HA, Chu WS, Yang DH, Hwang SD: Peritoneal accumulation of AGE and peritoneal membrane permeability. Perit Dial Int 2000;20:452-460.

29 Mandl-Weber S, Cohen CD, Haslinger B, Kretzler M, Sitter T: Vascular endothelial growth factor production and regulation in human peritoneal mesothelial cells. Kidney Int 2002;61:570-578.

30 Barre DE, Chen C, Cooker L, Moberly JB: Decreased in vitro formation of AGEs with extraneal solution compared to dextrose-containing peritoneal dialysis solutions. Adv Perit Dial 1999;15:12-16.

31 Lee JH, Reddy DK, Saran R, Moore HL, Twardowski ZJ, Nolph KD, Khanna R: Advanced glycosylation end-products in diabetic rats on peritoneal dialysis using various solutions. Perit Dial Int 2000;20:643-651.

32 Posthuma N, Verbrugh HA, Donker AJ, van Dorp W, Dekker HA, Peers EM, Oe PL, ter Wee PM: Peritoneal kinetics and mesothelia markers in CCPD using icodextrin for daytime dwell for two years. Perit Dial Int 2000;20: 174-180.

33 Millar DJ, Holmes C, Faict D, Dawnay A: Comparison of in vitro AGE formation between standard PD fluid and a novel bicarbonate/lactate formulation. Adv Perit Dial 1998; 14:191-194.

34 Park MS, Kim JK, Holmes C, Weiss MF: Effects of bicarbonate/lactate solution on peritoneal advanced glycosylation end-product accumulation. Perit Dial Int 2000;20(suppl 5):S33-S38.

35 Jonasson P, Braide M: A commercially available PD fluid with high $\mathrm{pH}$ and low GDP induces different morphological changes of rat peritoneum in intermittent PD. Adv Perit Dial 1998; 14:48-53.

36 Hekking LH, Zareie M, Driesprong BA, Faict D, Welten AG, de Greeuw I, Schadee-Eestermans IL, Havenith CE, van den Born J, ter Wee PM, Beelen RH: Better preservation of peritoneal morphologic features and defense in rats after long-term exposure to a bicarbonate/ lactate buffered solution. J Am Soc Nephrol 2001;12:2775-2786. 
37 Margetts PJ, Kolb M, Galt T, Hoff CM, Shockley TR, Gauldie J: Gene transfer of transforming growth factor-betal to the rat peritoneum: Effects on membrane function. J Am Soc Nephrol 2001;12:2029-2039.

38 Pertovaara L, Kaipanen A, Mustonen T, Orpana A, Ferrara N, Saksela O, Alitalo K: Vascular endothelial growth factor is induced in response to transforming growth factor-beta in fibroblastic and epithelial cells. J Biol Chem 1994;269:6271-6274.

39 Murohara T, Asahara T, Silver M, et al: Nitric oxide synthase modulates angiogenesis in response to tissue ischemia. J Clin Invest 1998; 101:2567-2578.

40 Papapetropoulos A, Garcia-Cardena G, Madri JA, Sessa WC: Nitric oxide production contributes to the angiogenic properties of vascular endothelial growth factor in human endothelial cells. J Clin Invest 1997;100:3131-3139.

41 Ziche M, Morbidelli L, Choudhuri R, et al: Nitric oxide synthase lies downstream from vascular endothelial growth factor-induced but not basic fibroblast growth factor-induced angiogenesis. J Clin Invest 1997;99:2625-2634.

42 Greten J, Kreis I, Weisel K, Stier E, Schmidt AM, Stern DM, Ritz E, Waldherr R, Nawroth PP: Receptors for advance glycation end-products (AGE)-expression by endothelial cells in non-diabetic uraemic patients. Nephrol Dial Transplant 1996;11:786-790.
43 Abel M, Ritthaler U, Zhang Y, Deng Y, Schmidt AM, Greten J, Sernau T, Wahl P, Andrassy K, Ritz E: Expression of receptors for advanced glycosylated end-products in renal disease. Nephrol Dial Transplant 1995;10: 1662-1667.

44 Boulanger E, Wautier MP, Wautier JL, Boval B, Panis Y, Wernert N, Danze PM, Dequiedt P: AGEs bind to mesothelial cells via RAGE and stimulate VCAM-1 expression. Kidney Int 2002;61:148-156.

45 Coles GA, O’Donoghue DJ, Pritchard N, Ogg CS, Jani FM, Gokal R, Cancarini GC, Maiorca R, Tranaeus A, De Vos C, Hopwood A, Faict D: A controlled trial of two bicarbonate-containing dialysis fluids for CAPD: Final report. Nephrol Dial Transplant 1998;13:3165-3171.

46 Feriani M, Kirchgessner J, La Greca G, Passlick-Deetjen J, Bicarbonate CAPD Cooperative Group: Randomized long-term evaluation of bicarbonate-buffered CAPD solution: Kidney Int 1998;54:1731-1738.

47 Lamb E, Cattell WR, Dawnay A: Glycated albumin in serum and dialysate of patients on continuous ambulatory peritoneal dialysis. Clin Sci (Colch) 1993;84:619-626.
48 Kjellstrand $\mathrm{P}$, Martinson E, Wieslander A, Holmquist B: Development of toxic degradation products during heat sterilization of glucose-containing fluids for peritoneal dialysis: influence of time and temperature. Perit Dial Int 1995;15:26-32.

49 Gotloib L, Bar-Sella P, Shostak A: Reduplicated basal lamina of small venules and mesothelium of human parietal peritoneum: Ultrastructural changes of reduplicated peritoneal basement membrane. Perit Dial Bull 1985;5: 212-215.

50 Dobbie JW, Lloyd JK, Gall CA: Categorization of ultrastructural changes in peritoneal mesothelium, stroma and blood vessels in uremia and CAPD patients. Adv Perit Dial 1990;6:312.

51 Churchill DN, Thorpe KE, Nolph KD, Keshaviah PR, Oreopoulos DG, Page D: Increased peritoneal membrane transport is associated with decreased patient and technique survival for continuous peritoneal dialysis patients. J Am Soc Nephrol 1998;9:1285-1292.

52 Krediet RT: The peritoneal membrane in chronic peritoneal dialysis. Kidney Int 1999; 55:341-356. 